\title{
The relationship of participation in shared governance to work satisfaction among cardiovascular nurses working in a tertiary hospital in Saudi Arabia
}

\author{
Naser Hussein Shwaihet ${ }^{* 1}$, Husain Ali Nasaif ${ }^{2}$ \\ ${ }^{1}$ King Faisal Specialist Hospital and Research Centre, Riyadh, Saudi Arabia \\ ${ }^{2}$ Royal College of Surgeon in Ireland - Bahrain, School of Nursing and Midwifery
}

Received: July 5, 2015

DOI: $10.5430 /$ cns.v3n4p79
Accepted: August 11, 2015

Online Published: August 30, 2015

URL: http://dx.doi.org/10.5430/cns.v3n4p79

\begin{abstract}
Shared governance has been used as a framework that provides nurses with both the structure and the mechanism to enhance decision-making authority and improve level of satisfaction. This study examined the level of work satisfaction among nurses working at cardiovascular department after the implementation of Shared Governance Model in a tertiary hospital in the Kingdom of Saudi Arabia. A descriptive cross sectional study design was adopted using the Index of Work Satisfaction Part-B (IWS-Part-B). Non-random convenience sample of 168 nurses who have worked in the cardiovascular department for more than two years received self-administered questionnaire (IWS-Part-B) on level of satisfaction about tasks, autonomy, interaction, decision making, professional status and pay. 145 nurses returned the questionnaire with a response rate of $86.3 \%$. Overall, nurses were moderately satisfied (IWS-Part-B: 181.10). They were moderately satisfied with autonomy, interaction, and professional status variables; while they were moderately unsatisfied with decision making, tasks requirements, and payment variables. Participation in decision making was the strongest predictor of work satisfaction, accounting for $71.4 \%$ of the explained variance $\left(r=0.845, R^{2}=0.714\right.$, $p=.000)$, followed by autonomy variable $\left(\mathrm{r}=0.821, \mathrm{R}^{2}=0.671, p=.000\right)$ and interaction variable $\left(\mathrm{r}=0.803, \mathrm{R}^{2}=0.645, p=\right.$ $.000)$. The findings of this study supported the positive role that shared governance plays in empowering nurses and enhancing their participation in the decision making process. Another multi-centers study is needed recruiting a larger sample from different departments that adopt SGM to better detect the effect of SGM on level of satisfaction and decision making in particular.
\end{abstract}

Key Words: Shared governance, Work satisfaction, Decision making, Nurses

\section{INTRODUCTION}

A shortage of nurses is a global problem that has affected many countries. ${ }^{[1]}$ There are several reasons for the worldwide shortage of nurses including demographic reasons, the availability of nursing education programs, poor work environment, inadequate compensation strategies, and work dissatisfaction..$^{[2]}$ Many nurses have cited an unhealthy work environment as the main reason for leaving a position. ${ }^{[3]}$
Nurses who report higher levels of work dissatisfaction are more likely to leave their workplaces. ${ }^{[4]}$ Many hospitals seeking magnet status have recognized this problem and have adopted different strategies to deal with it. Shared Governance Model (SGM) is one of the strategies that have been utilized to empower and improve nurses' working level of satisfaction in Magnet hospitals. Shared governance is "a dynamic process for achieving organizational effectiveness by

*Correspondence: Naser Hussein Shwaihet; Email: NShwaihet@kfshrc.edu.sa; Address: King Faisal Specialist Hospital and Research Centre, Riyadh, Saudi Arabia. 
promoting decision making and accountability for practice through empowerment", ${ }^{[5]}$ and is considered an integral component of Magnet hospitals and hospitals seeking Magnet recognition. It enhances communication and collaboration among the multidisciplinary team. ${ }^{[6]}$ The model offers nurses the opportunity to manage their own practice and influence decision making at managerial levels. ${ }^{[4]}$ Participation in the decision making improves nurses' satisfaction and decreases their intention to leave the profession. ${ }^{[7]}$

This study was conducted to contribute to the understanding of the relationship between nurses' participation in the decision making process as part of SGM and its impact on nurses' work satisfaction. Many studies have examined the relationship between SGM and work satisfaction. ${ }^{[8-15]}$ The findings, however, were not consistent and therefore could not be generalized into other contexts due to cultural variations and different characteristics of population. Some studies demonstrated that such nursing-led process was successful in achieving positive outcomes in patients' care and nurses' satisfaction. ${ }^{[10,16]}$ Other studies highlighted other contributing factors that could affect nurses' satisfaction including career development opportunities and supportive education. ${ }^{[11,17]}$

\subsection{Background}

The shortage of nurses in the Kingdom of Saudi Arabia (KSA) is acerbated by high turnover rates and the limited number of indigenous nurses with Saudi nurses representing only $29.1 \%$ of the total nursing workforce. ${ }^{[18]}$ Nursing turnover in KSA is considered a significant challenge that had impacted the efficiency of the health care system. ${ }^{[19]}$ The average nursing turnover rate in the hospital where this study took place was $12.5 \%$ during 2011 and $14 \%$ in 2010 . According to the hospital reports, personal and work-related factors were the main reasons for nurses' intention to leave the job. The SGM was adopted by the hospital and implemented in October 2010 as part of the Magnet recognition process to empower nurses, reduce turnover rate and deliver high effective care. Active involvement of nurses in the decision making process related to their practice is considered a key factor to the success of the SGM. ${ }^{[5]}$ A typical SGM consists of Unit Based Councils (UBCs) that are led by clinical nurses. ${ }^{[20]}$ UBCs collaborate together to enhance the practice within clinical areas. ${ }^{[20]}$

The model was updated by 2012 to provide clinical nurses with more opportunities for leading the decision making process. All nursing units have participated in the Magnet recognition program by having their own UBCs as part of the SGM. UBCs report to divisional councils from each nursing department. Representatives from divisional councils formulate the nursing affairs councils which direct nurses' practice in the hospital. They discuss issues, plans, and activities within the nursing affairs as provided by the UBCs. Member of UBCs have initiated several policies, protocols, and evidence-based guidelines. They also started to participate in different hospital wide committees and activities.

Several published studies have evaluated the effect of SGM on nurses' practice and satisfaction. ${ }^{[8,9,11,13,14,21,22]}$ In one study, the participation in shared governance enhanced the leadership skills of participants, but this was accompanied with many barriers. ${ }^{[8]}$ Two studies revealed improvement in the communication and collaboration among nurses who utilized SGM. ${ }^{[10,15]}$ Another study concluded that the involvement in shared governance was found to have a minor impact on nurses' participation in the decision making process when compared with other factors. ${ }^{[9]}$ Another study showed a negative correlation between shared governance and work satisfaction of nurses. ${ }^{[14]}$

The hospital where this study was conducted had participated in an international work satisfaction survey as part of the National Database of Nursing Quality Indicators during 2011. [23] The survey results revealed that cardiovascular nurses (CVN) were not satisfied with the ten satisfaction indicators (Tasks, autonomy, interaction, decision making, professional status, professional development, nursing management, nursing administration and pay). However, the survey was done few months after the implementation of SGM, and the results could not provide a reasonable reflection of the relationship between SGM and CVN work satisfaction level.

\subsection{Aim of the study}

This study aimed to examine the level of work satisfaction among nurses working in a cardiovascular department following the implementation of SGM. The study objectives were to examine the relationship between nurses' participation in the decision making process and the level of satisfaction and determine the level and the strongest predictors of work satisfaction. The research questions below were formulated to meet the aim and objectives.

\subsection{Research questions}

(1) What is the level of work satisfaction among CVNs after two years of SGM implementation?

(2) Is there a relationship between the demographic and personal characteristics and the level of work satisfaction among CVNs?

(3) What are the strongest predictors of work satisfaction among CVNs after SGM implementation? 


\section{MethodS}

This study was conducted over five month period between December 2012 and May 2013 in the cardiovascular department of a 936-bed tertiary hospital located in Riyadh-KSA. It is a Joint Commission International (JCI) accredited hospital providing advanced care for a wide range of patients including critical care, cardiac, oncology, and medical-surgical specialties. A quantitative, descriptive, cross-sectional and correlational design was used to guide the research process of the study. The study took place in seven units in the cardiovascular department. They were as follow: Cardiac Surgery Intensive Care Unit (CSICU), Cardiovascular Telemetry (CVT), Coronary Care Unit (CCU), Adult Medical Cardiology Unit (A4), Cardiovascular Step-down Unit (CVSD), Cardiac Catheterization Lab (Cath Lab) and Cardiology Lab-Noninvasive (Echo Lab).

The Index of Work Satisfaction Part B (IWS-Part-B) was used as the study tool. It is one of the most commonly used tools to measure work satisfaction of nurses. ${ }^{[24]}$ The Cronbach's Alpha coefficient of this tool is $0.70 .^{[24,25]}$ The IWS-Part B measures six variables: autonomy, decision making, interaction, professional status, task requirements, and pay. The IWS-Part-B included total of 44 items that were designed in a seven-option Likert scale where scale one indicated strongly agree and scale seven indicated strongly disagree. This scale had a neutral midpoint, scale 4 , which gave the opportunity for participants to distinguish between higher versus lower satisfaction levels. Half of the 44 items of the IWS-Part-B were positively phrased, and the other halves were negatively phrased. Those items, however, were randomly distributed in the tool. ${ }^{[26]}$ A pilot study was conducted including 17 nurses who had similar characteristics of the study sample.

There were approximately 271 nurses working in all the 7 units. A sample of nurses $(n=168)$ were recruited from cardiovascular units using non probability convenience sampling method. The sample included all nurses who have worked in the cardiovascular units for more than two years, before and after the implementation of SGM. Nurses who joined any of the cardiovascular units after the implementation of SGM in October 2010 were excluded from the study.

Ethical approval was obtained prior to the conduction of the study from the ethics and research committees of the Royal College of Surgeons in Ireland-Medical University of Bahrain and the Research Advisory Council (RAC) of the hospital where the study was conducted. A complete package including the questionnaire, study information sheet and consent form was sent to the participants who met the study inclusion criteria through the internal mailing system in the hospital. Personal data that might identify the study Published by Sciedu Press participants' were not obtained. The participants were given a period of two weeks to complete and submit the questionnaire. They were reminded at the end of the second week by sending them electronic reminders through the hospital emailing system. All completed questionnaires were returned in sealed envelopes.

\section{Statistical analysis}

The Statistical Package for Social Sciences (SPSS) computer software program for windows (Version 20.0) was used to analyze the data. Descriptive statistics for categorical variables were presented as a mean, frequency and percentage. Univariate analysis comparing the significance differences in the mean score of level of satisfaction using paired t-test and Kruskal Wallis test was performed. Correlations coefficient test was used to examine the relationship between the level of satisfaction and main tool's six variables.

\section{FINDINGS}

The sample consisted of 168 nurses from seven cardiovascular units. A total of 145 respondents returned the study self-administered questionnaire with an overall response rate of $86.3 \%$. CSICU and Echo Lab had $100 \%$ response rate. CVT had the lowest response rate $(70.4 \%)$

\subsection{Demographic \& personal characteristics}

Sample demographics and personal characteristics are summarized in Table 1. Respondents' age ranged between 20-60 years. Overall, $57.9 \%$ of respondents were at age group between $31-40$ years old and $76 \%$ were females. 89 respondents $(61.4 \%)$ reported combined shifts (day and night) as their regular working shift, and 56 respondents (38.6\%) reported one permanent working shift (Day shift, night shift, or business hours: 07:30 am-5:00 pm). 63\% of respondents held Bachelor degree in Nursing and $44.1 \%$ had working experience less than 5 years in the current unit.

\subsection{Level of work satisfaction}

Quartile rankings were utilized to determine the classification of the level of satisfaction about each variable of IWS-Part B tool (Quartile 1: unsatisfied, Quartile 2: moderately unsatisfied, Quartile 3: moderately satisfied and Quartile 4: satisfied). ${ }^{[27]}$ Each respondent's level of satisfaction was calculated by taking the sum of the scorings of the 44 statements included in the IWS-Part B tool (range: 44-308). The overall CVNs score of the IWS-Part B was 181.1 (Lower Limit: 176.3, Upper Limit: 185.7, Confidence Interval 95\%). The level of work satisfaction by cardiovascular units was summarized in Table 2. Nurses working in Echo Lab, CCU, and CVSD were moderately satisfied, while nurses working in CSICU, A4, CVT, and Cath Lab were moderately unsatisfied. 
Table 1. Sample demographics \& personal characteristics

\begin{tabular}{|c|c|c|}
\hline Variable & Frequency & Percentage (\%) \\
\hline \multicolumn{3}{|l|}{ Gender } \\
\hline Male & 35 & 24.1 \\
\hline Female & 110 & 75.9 \\
\hline Total & 135 & 100 \\
\hline \multicolumn{3}{|l|}{ Age } \\
\hline $20-30 Y$ & 24 & 16.6 \\
\hline $31-40 Y$ & 84 & 57.9 \\
\hline $41-50 Y$ & 25 & 17.2 \\
\hline $51-60 Y$ & 12 & 8.3 \\
\hline Total & 145 & 100 \\
\hline \multicolumn{3}{|l|}{ Working Shift } \\
\hline 7 AM -7 PM & 20 & 13.8 \\
\hline 7 PM-7 AM & 20 & 13.8 \\
\hline Both & 88 & 60.7 \\
\hline 7:30 AM-5 PM & 17 & 11.7 \\
\hline Total & 145 & 100.0 \\
\hline \multicolumn{3}{|c|}{ Total Years of Experience in Current Unit } \\
\hline $0-5 Y$ & 64 & 44.1 \\
\hline $5-10 Y$ & 53 & 36.6 \\
\hline $10-15 Y$ & 24 & 16.6 \\
\hline $16-20 Y$ & 2 & 1.4 \\
\hline $21-30 Y$ & 2 & 1.4 \\
\hline Total & 145 & 100.0 \\
\hline \multicolumn{3}{|c|}{ Total Years of Experience in Nursing Profession } \\
\hline $0-5 \mathrm{Y}$ & 9 & 6.2 \\
\hline $5-10 Y$ & 43 & 29.7 \\
\hline $10-15 Y$ & 48 & 33.1 \\
\hline $16-20 Y$ & 28 & 19.3 \\
\hline $21-30 Y$ & 14 & 9.7 \\
\hline$+31 \mathrm{Y}$ & 3 & 2.1 \\
\hline Total & 145 & 100.0 \\
\hline \multicolumn{3}{|c|}{ Degree of Academic Education } \\
\hline Diploma in Nursing & 48 & 33.1 \\
\hline Associate in Nursing & 5 & 3.4 \\
\hline Bachelor in Nursing & 91 & 62.8 \\
\hline Other & 1 & 0.7 \\
\hline Total & 145 & 100.0 \\
\hline
\end{tabular}

\subsection{Predictors of work satisfaction}

The level of satisfaction according to the IWS-Part B six variables were summarized in Table 3. CVNs were moderately satisfied (Quartile 3) with autonomy, interaction (nurse-nurse and nurse-physician), and professional status, however, they were moderately unsatisfied with decision making, tasks, and pay. The relationship between the level of satisfaction and the six variables were also examined (see Table 4). The most significant and strongest positive correlation was found between work satisfaction and decision making $(\mathrm{r}=0.845, p=$ $.000)$ followed by autonomy and interaction variables. Work satisfaction had a moderately positive correlation with professional status, tasks and payment variables. Relationships between the variables were also explored using simple linear regression tests. According to this statistical test, $71.4 \%$ of the variations in the work satisfaction level were attributed to the decision making variable $\left(\mathrm{R}^{2}=0.714, p=.000\right)$. Also, $67.1 \%$ of the variations in the work satisfaction level were attributed to the autonomy variable $\left(\mathrm{R}^{2}=0.671, p=.000\right)$, and $64.5 \%$ of the variations in the work satisfaction level were related to the interaction variable $\left(\mathrm{R}^{2}=0.645, p=0.000\right)$. 
Table 2. Level of satisfaction by units (Quartiles and rankings of the IWS-Part-B)

\begin{tabular}{llll}
\hline Unit & IWS-Part B Score & Ranking & Quartile \\
\hline Echo Lab & 210.60 & Moderately Satisfied & Quartile 3 \\
CCU & 186.55 & Moderately Satisfied & Quartile 3 \\
CVSD & 183.72 & Moderately Satisfied & Quartile 3 \\
CSICU & 179.53 & Moderately Unsatisfied & Quartile 2 \\
A4 & 178.85 & Moderately Unsatisfied & Quartile 2 \\
CVT & 174.89 & Moderately Unsatisfied & Quartile 2 \\
Cath Lab & 163.57 & Moderately Unsatisfied & Quartile 2 \\
\hline
\end{tabular}

\subsection{Difference in the mean score of work satisfaction}

The relationship between the level of work satisfaction and the demographic and personal characteristics were examined (see Table 5). There was no significant differences in the mean score of work satisfaction in relation with gender $(p=$ .581), however, male was more satisfied than female (mean $=21.47, p=.034)$ with Nurse-Physician Interaction.

Table 3. Level of satisfaction according to the IWS-Part-B six variables (Quartiles and Rankings)

\begin{tabular}{llll}
\hline Variable & Score & Ranking & Quartile \\
\hline Overall level & 181.4 & Moderately satisfied & Quartile 3 \\
Autonomy & 34.34 & Moderately Satisfied & Quartile 3 \\
Interaction & 43.39 & Moderately Satisfied & Quartile 3 \\
Professional Status & 35.75 & Moderately Satisfied & Quartile 3 \\
Decision Making & 27.68 & Moderately Unsatisfied & Quartile 2 \\
Tasks & 23.36 & Moderately Unsatisfied & Quartile 2 \\
Pay & 16.53 & Moderately Unsatisfied & Quartile 2 \\
\hline
\end{tabular}

In relation to age, the study found that there was no significant differences in the mean score of work satisfaction in ( $p$ $=.140)$. However, age had a statistically significant relationship with the interaction variable $(p=.048)$. Nurses with an age range of 51-60 years had the highest level of satisfaction with the interaction variable (mean $=49.08$ ), while nurses with an age range of 41-50 years had the lowest level of satisfaction (mean $=42.28$ ). Similarly, a statistically significant relationship was found between age and nurse-nurse interaction $(p=.039)$. The 51-60 age group had the highest level of satisfaction (mean $=26.42$ ), and the 41-50 age group had the lowest satisfaction level (mean $=22.92$ ). Total years of experience in current unit had a significant relationship with the nurse-nurse interaction variable $(p=.037)$. Nurses who had worked between 16-20 years in their current unit had the highest level of satisfaction (mean $=27.50$ ), while nurses with 10-15 years of experience in their current unit had the lowest level $($ mean $=21.83)$.

Nurses with diploma degree were more satisfied (IWS-PartB: 183.5 , moderately satisfied) in comparison with bachelor degree holders (IWS-Part-B: 178.3, moderately unsatisfied). Nurses with bachelor degree were more satisfied with the interaction variable (43.51, moderately satisfied) in comparison with the nurses who had diploma degree (42.67, moderately Published by Sciedu Press satisfied). However, there were no significant differences ( $p$ $=.438$ ). Nurses who worked day duty had higher levels of work satisfaction (IWS-Part-B: 189.2, moderately satisfied) in comparison with those who worked night duty (IWS-PartB: 175.3, moderately unsatisfied) and those who worked combined shifts (IWS-Part-B: 179.4, moderately unsatisfied). However, the difference was not significant $(p=.136)$.

\section{Discussion}

This is the first study conducted in Saudi Arabia that examined the level of satisfaction following the implementation of SGM as part of magnet status accreditation. This topic was selected because the hospital adopted SGM for more than two years to improve nurses' satisfaction and participation in the decision making process; however, this relationship had not been thoroughly evaluated following implementation. Several methods were utilized to ensure adequate responses from participants. The study was presented to the CVNs during their council meetings one month prior to the data collection process. An email explaining the purpose of this study was also sent to all CVNs by the director of the hospital nursing research council. Electronic reminders were also sent to all potential participants after two weeks from sending the study tool. 
Table 4. Dependent variables correlation coefficient

\begin{tabular}{llllllll}
\hline Variable & Work satisfaction & Autonomy & Interaction & Decision making & Professional status & Tasks & Pay \\
\hline Pearson correlation & 1 & $0.821^{*}$ & $0.803^{*}$ & $0.845^{*}$ & $0.670^{*}$ & $0.655^{*}$ & $0.507^{*}$ \\
Sig. (2-tailed) & & .000 & .000 & .000 & .000 & .000 & .000 \\
\hline
\end{tabular}

* Correlation is significant at the 0.01 level (2-tailed)

The main finding of this study was that CVNs were overall moderately satisfied (Quartile 3: 181.1, Lower Limit: 176.3, Upper Limit: 185.7, Confidence Interval 95\%). This finding was different from the 2011 study that was carried out five months after the implementation of SGM. Cardiovascular nurses were dissatisfied with the ten satisfaction indicators in the 2011 survey (Tasks, autonomy, interaction, decision making, professional status, professional development, nursing management, nursing administration and pay). This finding is consistent with many other studies which revealed that the participation in shared governance as part of the Magnet program has enhanced nurses' level of satisfaction. ${ }^{[6,10,21,28-33]}$

Table 5. Level of work satisfaction and the demographic and personal characteristics

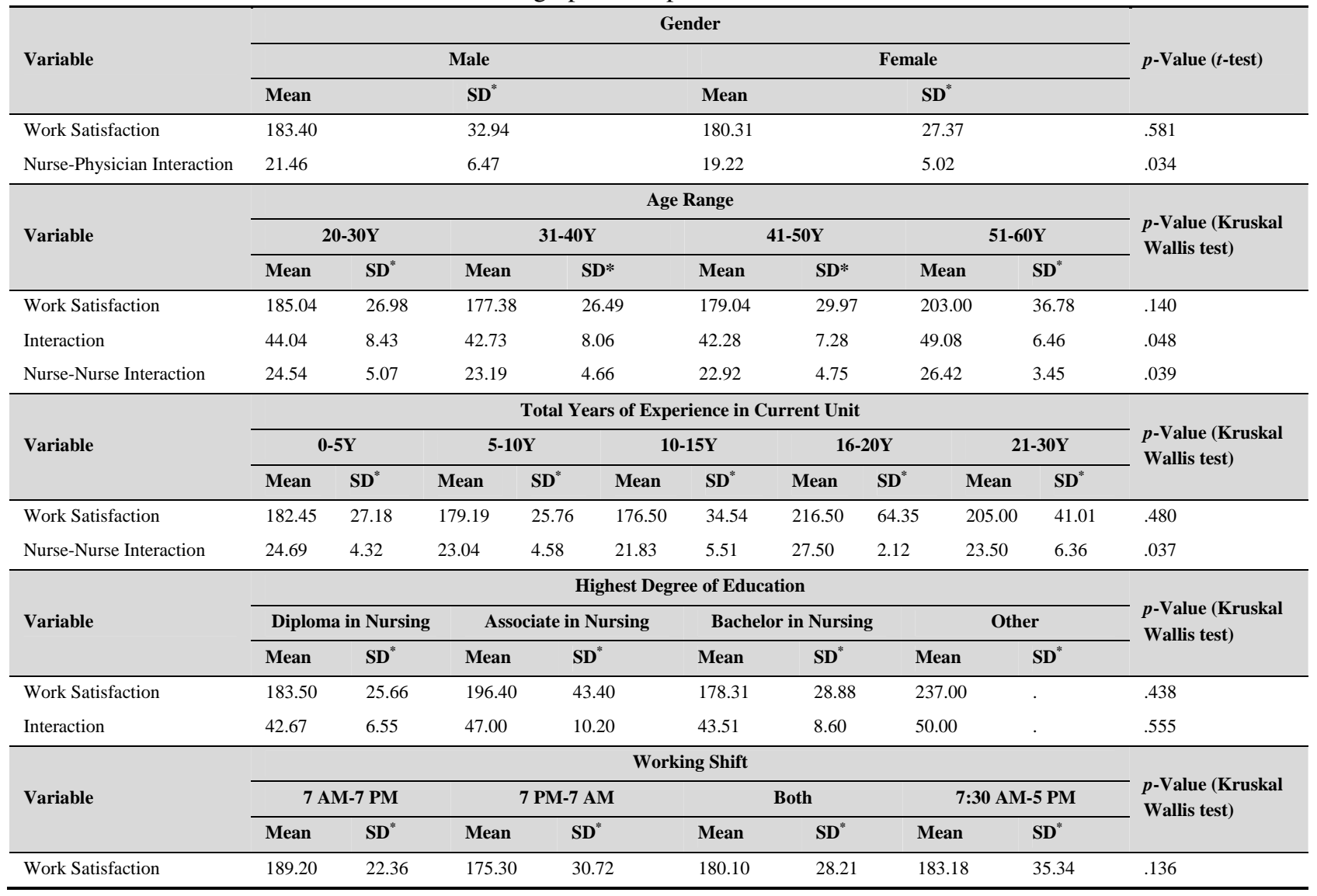

* SD = Standard Deviation

CVNs were moderately satisfied with autonomy, interaction, and professional status variables. Enhanced level of autonomy is one the main benefits of the participation in SGM. ${ }^{[4]}$ CVNs interaction level had improved between and among nurses and between nurses and physicians. This finding supports the empirical evidence in the literature regarding the positive correlation between participation in the decision making and the communication within the multi- disciplinary team. ${ }^{[28,31]}$ The leadership team of the hospital where this study was carried out has implemented different strategies to involve the medical team in the UBCs activities in their individual units. To date; many physicians have participated in the UBC meetings of the cardiovascular units. The increased level of CVNs satisfaction with their professional status should be interpreted in light of different initiatives and strategies that have been implemented with 
the aim of recognizing the contribution of nurses to health care services within the hospital. The initiatives included sponsoring nurses for higher education studies; Saudi nurses in particular, encouraging nurses to attend scientific national and international conferences, engaging nurses in different hospital-wide projects, and paying the costs of nurses' specialty certification exams.

Several studies have indicated that the SGM has improved nurses' participation in the decision making process. ${ }^{[9,11,12,28,29]}$ Interestingly, in this study CVNs were still unsatisfied with decision making processes two years following the implementation of SGM. This finding could be attributed to some factors and potential limitations. Firstly, the SGM was incorporated within nurses' practice by the end of 2010 when nurses were not fully engaged with the new model of practice. Secondly, the structure of SGM has gone through several changes following implementation in 2010. Thirdly, there were no proper training programs in the hospital to orient nurses on the aspects of SGM.

CVNs were moderately unsatisfied with the tasks variable. They were also unsatisfied with this variable in the survey that was conducted in the hospital during 2011. ${ }^{[23]}$ Though the SGM is considered a new model for CVNs, its impact on their practice and daily activities are not well well-reflected from their perspective. Even though many policies and practices were revised by the nurses themselves after SGM implementation, the effect of those changes may require a longer time span to reflect on their level of satisfaction and task performance. CVNs were also moderately unsatisfied with their pay scales. This finding was similar to the results of the study that was conducted in the hospital during 2011. ${ }^{[23]}$ There are many hospitals in the Arabian Gulf region that pay nurses compatible salaries and reimbursements. The presence of low payments and lack of rewards are considered the main contributing factors to the decreased levels of nurses' satisfaction. $^{[34]}$

The study findings revealed a statistically significant relationship between gender and the nurse-physician interaction variable. Male nurses had a higher level of satisfaction with their relationship with physicians in comparison with female nurses. This finding was attributed mainly to the cultural context within the country where the study was conducted. It is well-known that the culture in KSA is a male-dominant culture. ${ }^{[35]}$ Most of the male nurses in the cardiovascular division were from neighboring Arabic countries, while the majority of the female nurses were from Asian countries. Nurses with an Arabic background had a better understanding of the cultural considerations within the Arabian Gulf region. ${ }^{[35]}$ In addition, a statistically significant relationship was found between age and the interaction variable. Nurses with an age range of 51-60 years had the highest level of satisfaction with the interaction variable. $12 \mathrm{CVN}$ comprised this age group. The interaction variable was sub-divided into two main variables: nurse-nurse interaction and nurse-physician interaction. There was a significant relationship between age and nurse-nurse interaction. Older nurses typically have more experience working with different team members for a longer time than younger nurses. Time factor is crucial for nurses to be exposed to different communication styles with physicians and other health care professionals. ${ }^{[8]}$

Years of experience in the current unit had a statistically significant relationship with the nurse-nurse interaction variable. CVNs who had worked in their units for more than 15 years had the highest level of satisfaction with the nurse-nurse interaction variable. In fact, nurses with more years of experience had the highest overall level of satisfaction and satisfaction with autonomy, interaction, decision making, professional status, tasks, and pay variables. Those findings were similar to the results of several published research studies that had examined the relationship between the years of experience and the level of work satisfaction. ${ }^{[33,36]}$ CVNs with more years of experience in their units were exposed to different communication styles with other health care professionals. Another study also supported this finding and concluded that nurses with more than 30 years of experience had higher levels of satisfaction. ${ }^{[33]}$

\section{Limitation \& future recommendation}

SGM was implemented in all departments of the hospital. However, this study had only examined the level of satisfaction among nurses working in cardiovascular department. Therefore, the findings cannot be generalized to nurses working in other departments. Another limitation of the study was that other confounding factors like nurse-patient ratio, marital status, and nationality were not controlled. These confounding factors may have affected the outcomes of the study.

Studying the relationship between SGM and work satisfaction did not consider other factors that could affect the study results including the duration of SGM implementation, the involvement in advanced technologies within the clinical areas, the participation in other accreditation processes such as JCI Accreditation, and the expansion of services within the organization. Using mailed surveys inhibited the researchers from controlling any extraneous variables that could affect the level of work satisfaction such as the time when the study was conducted. The hospital decided to amend the rules and regulations of the SGM during December 2012. This occurred during the data collection process, which could have 
had a negative impact on the outcome of the study.

The inclusion of patients' acuity and nurse-patient ratio should be considered in future researches. Inclusion of more variables such as marital status and nationality could provide better correlation between participants' demographics and the study variables. Comparing the relationship between the study variables in more than one hospital would provide a more realistic reflection regarding the relationship between those variables. Future studies should consider the period of shared governance implementation. Studying the impact of SGM on nurses' level of work satisfaction could be more objectively measured after providing adequate time for nurse to incorporate the concepts of SGM within their practice. Longitudinal research, before and after SGM implementation, would provide more valuable information about the relationship between SGM and work satisfaction. Using more than one study instrument such as work environment scale could assist in providing more reliable information about the relationship between the different variables. Measuring the effectiveness of UBCs through specific tools could provide more valuable information regarding the efficiency of SGM. In addition, qualitative methodology should be considered in future researches.

\section{Conclusion}

Participation in the decision making process as supported by the shared governance model has been cited as a strategy to enhance nurses' work satisfaction. This study contributes to the understanding of the relationship between nurses' participation in the decision making process as part of SGM and its impact on nurses' work satisfaction. It also provides guidance for hospitals and nursing administrators toward effective implementation of SGM.

The results of this study supported the findings of previous studies which made a positive correlation between the participation in shared governance and the work satisfaction of nurses. Though cardiovascular nurses were more satisfied in comparison with the study that was conducted in their hospital during 2011, they were moderately unsatisfied with their participation in decision making process, the tasks they provide, and the payments they receive. This is an indicator that nurses' involvement in the shared governance to improve their level of work satisfaction should consider other contributing factors including the duration of participation, nurses' workload, and the presence of satisfactory reimbursement systems. The success of SGM requires longer time to reflect on nurses' practice. Future studies could provide better correlation between nurses' involvement in the decision making process as part of the SGM and their actual level of work satisfaction.

\section{ACKNOWLEDGEMENTS}

This study was done and submitted in part fulfillment of the degree of Master in Sciences in Nursing, Faculty of Nursing \& Midwifery, Royal College of Surgeon in Ireland - Medical university of Bahrain, 2013. Special thanks to the persons who have assisted in completing this study including Dr. Catherine S. O'Neill, the director of MSc Nursing Program at Royal College of Surgeon in Ireland - Medical University of Bahrain, Dr. Mohammed Shoukri, Mrs. Maya Sinno, Mr. Mohammad Al-Hawari, Mrs. Fatima Alzu'bi and Mr. Mohammed Dagamseh from the King Faisal Specialist Hospital and Research Centre, Riydh Saudi Arabia.

\section{REFERENCES}

[1] Mullett S. Nursing shortage expected to worsen through 2016. Healthcare Traveler. 2009; 16(11): 24.

[2] Fox R, Abrahamson K. A critical examination of the U.S. nursing shortage: contributing factors, public policy implications. Nursing Forum. 2009; 44(4): 235-244. http://dx.doi.org/10.1111/j $.1744-6198.2009 .00149 . x$

[3] Hassmiller S, Cozine M. Essay. Addressing the nurse shortage to improve the quality of patient care: efforts of the RWJF and selected funders around the nation to fix this problem affecting today's hospitals. Health Affairs. 2006; 25(1): 268-274.

[4] Roach EJ. Shared governance in nursing. Nursing Journal of India. 2009; 100(4): 76-78. PMID:20527491.

[5] Bednarski D. Shared governance: enhancing nursing practice. Nephrology Nursing Journal. 2009; 36(6): 585. PMID:20050511.

[6] Gaguski M, Begyn P. A unique model of shared governance. Oncology Nursing Forum. 2009; 36(4): 385-388. http://dx. doi .org /10.1188/09.0NF.385-388
[7] Zoschak EW. Indicators of excellent nursing care. Trustee. 2010; 63(9): 28-30. PMID:21090038.

[8] Williamson T. Work-based learning: a leadership development example from an action research study of shared governance implementation. Journal of Nursing Management. 2005 Nov; 13(6): 490-9. PMID:16238690. http://dx.doi.org/10.1111/j.1365-2934. 2005.00576.x

[9] Kent V. Rondeau. The adoption of high involvement work practices in Canadian nursing homes. Leadership in Health Services. 2007; 20(1): 16-26. http://dx.doi.org/10.1108/17511870710721453

[10] Ulrich B, Woods D, Hart K, et al. Critical care nurses' work environments: value of excellence in Beacon units and Magnet organizations. Critical Care Nurse. 2007; 27(3): 68-77.

[11] Wilson B, Squires M, Widger K, et al. Job satisfaction among a multigenerational nursing workforce. Journal Of Nursing Management. 2008; 16(6): 716-723. http://dx.doi.org/10.1111/j.1365-2 $834.2008 .00874 . \mathrm{x}$

[12] Fray B. Evaluating shared governance: measuring functionality of 
unit practice councils at the point of care. Creative Nursing. 2011; 17(2): 87-95. PMID:21563636. http://dx . doi .org/10.1891/1 078-4535.17.2.87

[13] Abdelkader R, Mahmoud OA, Barmawi MA, et al. Perception of academic nursing staff toward shared governance. Journal of Nursing Education and Practice. 2012; 2(3): 46. http://dx.doi.org/10. $5430 /$ jnep.v2n3p46

[14] McGLYNN K, Griffin M, Donahue M, et al. Registered nurse job satisfaction and satisfaction with the professional practice model. Journal Of Nursing Management. 2012; 20(2): 260-265. http: //dx.doi.org/10.1111/j.1365-2834.2011.01351.x

[15] Sakowski P. Job satisfaction of occupational medicine nurses in Poland. International Journal Of Occupational Medicine \& Environmental Health. 2012; 25(1): 51-58.

[16] Johnson K, Hallsey D, Meredith R, et al. A nurse-driven system for improving patient quality outcomes. Journal of Nursing Care Quality. 2006; 21(2): 168-175.

[17] Ernst M, Franco M, Messmer P, et al. Practice applications of research. Nurses' job satisfaction, stress, and recognition in a pediatric setting. Pediatric Nursing. 2004; 30(3): 219-227.

[18] Almalki M, FitzGerald G, Clark M. The nursing profession in Saudi Arabia: an overview. International Nursing Review. 2011; 58(3): 304-311. http://dx.doi.org/10.1111/j.1466-7657. $2011.00890 . x$

[19] Walston S, Al-Harbi Y, Al-Omar B. The changing face of healthcare in Saudi Arabia. Annals Of Saudi Medicine. 2008; 28(4): 243-250.

[20] Pierson MA, Liggett C, Moore KS. Twenty years of experience with a clinical ladder: A tool for professional growth, evidence-based practice, recruitment, and retention. The Journal of Continuing Education in Nursing. 2010; 41(1): 33-40. http://dx.doi.org/10.3928/0 0220124-20091222-06

[21] Ulrich B, Lavandero R, Hart K, et al. Healthy work environments. Critical care nurses' work environments: a baseline status report. Critical Care Nurse. 2006; 26(5): 46.

[22] Sakowski J, Hooper L, Holton T, et al. Partners Advancing Clinical Excellence: Building Professional Councils for Quality Improvement at Six Community Hospitals. Creative Nursing. 2012; 18(4): 177-186.

[23] King Faisal Specialist Hospital and Research Center-KFSH\&RC. RN survey results. 2011.
[24] Zangaro G, Soeken K. Meta-analysis of the reliability and validity of part B of the index of work satisfaction across studies. Journal Of Nursing Measurement. 2005; 13(1): 7-22.

[25] Anthony D. Understanding Advanced Statistics: A Guide for Nurses and Healthcare Researchers. Churchill Livingstone: Edinburgh; 1999.

[26] Stamps PL. Scoring Workbook for the Index of Work Satisfaction. Market Street Research: Northampton, MA; 1977.

[27] Stamps PL. Direction for Decisions, Scoring Workbook for the Index of Work Satisfaction. Market Street Research: Northampton, MA; 2001.

[28] Laschinger H, Shamian J, Thomson D. Impact of magnet hospital characteristics on nurses' perceptions of trust, burnout, quality of care, and work satisfaction. Nursing Economics. 2001; 19(5): 209-219.

[29] Rondeau K, Wagar T. Nurse and resident satisfaction in magnet long-term care organizations: do high involvement approaches matter? Journal Of Nursing Management. 2006; 14(3): 244-250. http://dx.doi.org/10.1111/j.1365-2934.2006.00594.x

[30] Jae SP, Tae HK. Do types of organizational culture matter in nurse job satisfaction and turnover intention? 2009; 22(1): 20-38. http: //dx.doi.org/10.1108/17511870910928001

[31] McArthur A. Review summaries: evidence for nursing practice. A comprehensive systematic review of evidence on determining the impact of Magnet designation on nursing and patient outcomes: is the investment worth it? Journal of Advanced Nursing. 2010; 66(6): 1211-1212. http://dx.doi.org/10.1111/j.1365-2648. 2010 $.5309 . x$

[32] Graham K, Davies B, Woodend A, et al. Impacting Canadian public health nurses' job satisfaction. Canadian Journal of Public Health. 2011; 102(6): 427-431.

[33] Kramer M, Maguire P, Brewer B. Clinical nurses in magnet hospitals confirm productive, healthy unit work environments. Journal Of Nursing Management. 2011; 19(1): 5-17. http://dx.doi.org/1 $0.1111 / j .1365-2834.2010 .01211 . x$

[34] Pillay R. Retention strategies for professional nurses in South Africa, Leadership in Health Services. 2009; 22(1): 39-57.

[35] Shah M, Al-Enezi N, Chowdhury R, et al. Determinants of job satisfaction among nurses in Kuwait. Australian Journal Of Advanced Nursing. 2004; 21(4): 10-16.

[36] Atencio B, Cohen J, Gorenberg B. Nurse retention: is it worth it? Nursing Economics. 2003; 21(6): 262. 